\title{
L'Ordre et la morale : quand l'industrie du cinéma bouscule la coutume kanak
}

\section{Mathias FAURIE et Mélissa NAYRAL}

\section{(2) OpenEdition \\ 12 Journals}

Édition électronique

URL : http://journals.openedition.org/jso/6641

DOI : $10.4000 /$ jso. 6641

ISSN : $1760-7256$

Éditeur

Société des océanistes

Édition imprimée

Date de publication : 30 juin 2012

Pagination : 121-136

ISBN : 978-2-85430-032-1

ISSN : 0300-953x

Référence électronique

Mathias FAURIE et Mélissa NAYRAL, «L'Ordre et la morale : quand l'industrie du cinéma bouscule la coutume kanak », Journal de la Société des Océanistes [En ligne], 134 | 1er semestre 2012, mis en ligne le 12 juillet 2012, consulté le 19 avril 2019. URL : http://journals.openedition.org/jso/6641 ; DOI : 10.4000/jso.6641 


\title{
L'Ordre et la morale: quand l'industrie du cinéma bouscule la coutume kanak
}

par

\author{
Mathias FAURIE et Mélissa NAYRAL*
}

Le film L'Ordre et la morale, réalisé par Mathieu Kassovitz, réalisateur et acteur désormais incontournable du cinéma français, relatant la prise d'otages au dénouement tragique de 1988 à Ouvéa (Nouvelle-Calédonie), est sorti en salle le 16 novembre 2011. Tout au long de sa production, il s'est trouvé au cœur d'importantes polémiques suscitant de vives prises de positions médiatiques ${ }^{1}$ qui auguraient déjà la suite : alors qu'une importante campagne de promotion était à l'œuvre en métropole pour préparer la sortie nationale, le film se trouvait censuré par le principal patron de salles de cinéma en NouvelleCalédonie $^{2}$. Pour Douglas Hickson, patron du Cinecity de Nouméa, L'Ordre et la morale est « très caricatural » et il « rouvre des plaies qui s'étaient cicatrisées » (AFP, 20/10/2011).

L'affaire d'Ouvéa » ou « affaire de la grotte », paroxysme de la crise sociopolitique des « événements ", semble toujours attachée à ce drame humain et politique enfoui sous le silence d'une amnistie générale. Au-delà de cette image, elle constitue depuis 1988 un nœud historique de la Nouvelle-Calédonie contemporaine et demeure visiblement un sujet brûlant autour duquel se cristallisent les problématiques à venir en terme de construction identitaire et territoriale. La difficulté de certains, tant calédoniens que français, à assumer ce que d'autres nomment « une faillite de la République » (Drevillon, 2008), nous rappelle combien l'histoire de la Nouvelle-
Calédonie de ces deux derniers siècles est jalonnée de drames, d'injustices et d'incompréhensions. Plus de vingt années après l'affaire d'Ouvéa qui déboucha sur les accords de Matignon (1988), force est de constater la permanence de grands clivages sociétaux et la fragilité de la communauté de destin ${ }^{3}$ projetée par l'accord de Nouméa (1998). Toutefois, le dialogue et la réflexion sur l'histoire du territoire permettent peu à peu, malgré des mouvements réactionnaires et une certaine censure, de construire cette appartenance partagée et d'amener à la réconciliation entre les familles et les communautés.

Ce film arrive ainsi à point nommé pour réactiver le débat sur l'avenir de la NouvelleCalédonie et rafraîchir la mémoire, en particulier celle des métropolitains, sur le passé peu glorieux de la « France des cocotiers ». Avec un budget de l'ordre de 15 millions d'euros qui pulvérise les records locaux, ce film est le plus gros projet cinématographique jamais réalisé en NouvelleCalédonie. Son sujet est difficile et très ambitieux, compte tenu du traumatisme que représentent localement « les événements d'Ouvéa ». L'Ordre et la morale s'est donné pour objectif de raconter, le plus justement possible, les quatorze jours entre le 22 avril 1988 , jour de la prise d'otage des gendarmes de la brigade de Fajawe ${ }^{4}$, et le 5 mai 1988 qui marque la fin de « l'Opération Victor » à la grotte de Goosana.

1. Notamment par des personnalités politiques locales telles que P. Gomes, R. Wamytan, P. Frogier etc.; cf. Les Nouvelles calédoniennes du 30/10/2011.

2. Nouvelle-Calédonie Première du 21/10/2011, l'Express du 22/10/2011, Le Monde du 23/10/2011, Libération du 21/10/2011, etc.

3. L'accord de Nouméa entérine le principe du « destin commun » pour les communautés résidant sur le territoire.

4. Les toponymes locaux sont écrits en langue iaai ou fagauvea.

* Docteur en géographie de l'Université Paris 4 Sorbonne- PRODIG, mathiasfaurie@gmail.com, doctorante en anthropologie à l’Université de Provence-CREDO, melissa.nayral@gmail.com 


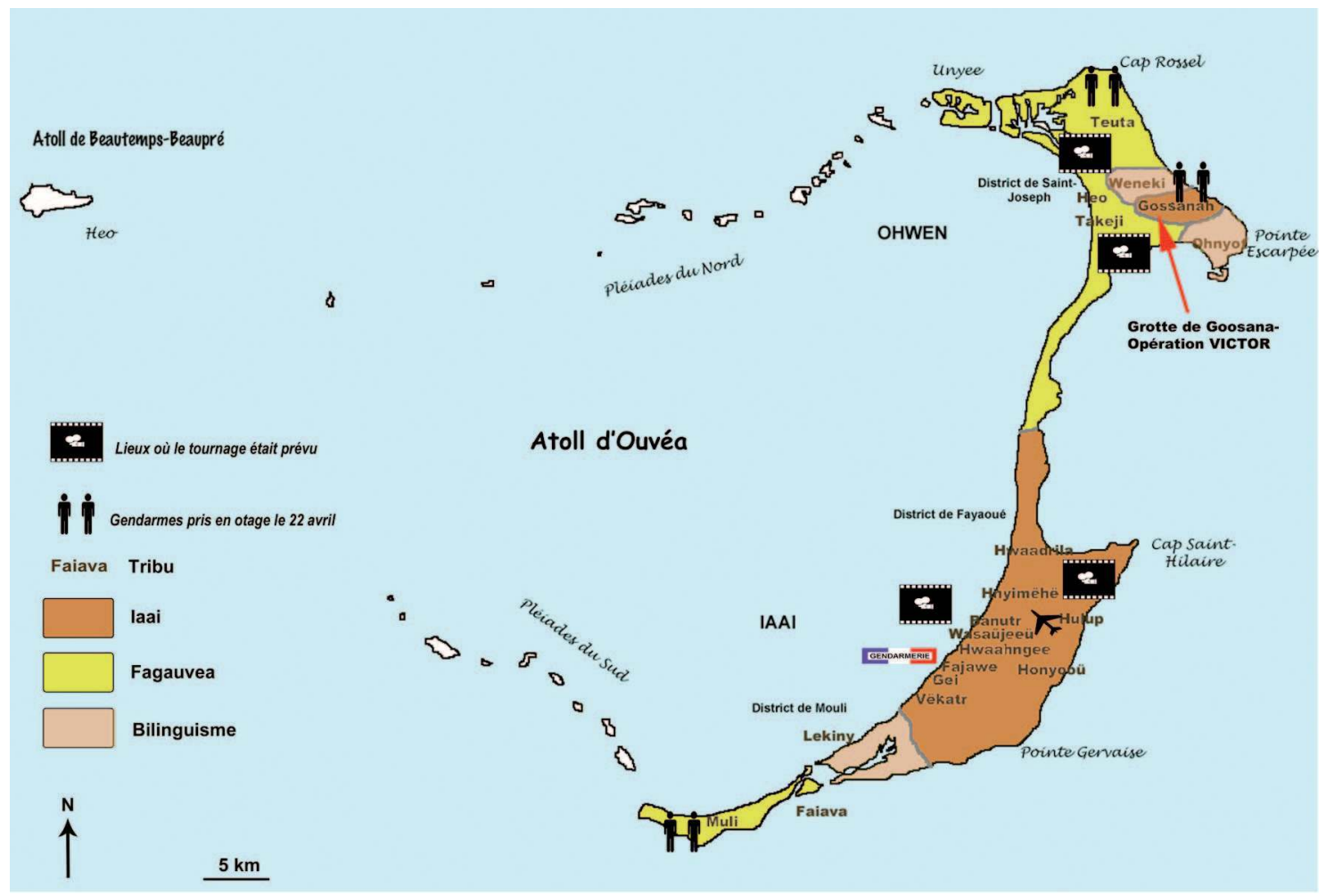

CARTE 1. - Ouvéa : Organisation de l'espace, événements de 1988 et projet de tournage du film (carte réalisée par M. Faurie)

En ce sens, il ne relève pas du genre cinématographique qu'on appelle cinéma-vérité, mais des genres « Histoire » et « Action » des classifications habituelles des grandes productions. Ce n'est ni un documentaire ${ }^{5}$ focalisant sur un point particulier de la réalité, ni un docu-fiction qui la copierait dans ses moindres détails. Au moyen d'un casting et d'un tournage un peu particuliers, le réalisateur souhaitait que la population d'Ouvéa soit au premier plan et s'implique activement dans le projet y compris en tant qu'acteurs. Pour ce faire, le tournage devait initialement avoir lieu sur l'atoll et être pleinement porté par sa population. Le film a finalement pu être tourné, mais en Polynésie française. Comme d'autres grands projets relatifs à la NouvelleCalédonie et à Ouvéa en particulier, il a connu des difficultés majeures qui, loin d'être anecdotiques, sont au contraire révélatrices d'une société complexe et fragmentée où les jeux de pouvoirs et les divisions internes expliquent en grande partie les blocages et les inerties ${ }^{6}$. À l'analyse, il apparaît clairement que les enjeux qui se sont trouvés cachés derrière ce projet et les complica- tions qu'il a engendrées, ou auxquelles les cinéastes ont dû faire face, ont cristallisé des problèmes qui existent à l'échelle de la Nouvelle-Calédonie tout entière.

Lors de nos travaux de terrain sur l'île d'Ouvéa dans le cadre de nos recherches de doctorat $^{7}$ en géographie et anthropologie, nous avons été confrontés à la mise en place de ce projet qui a constitué non seulement une expérience tout à fait originale pour la population d'Ouvéa et pour nous, mais qui a révélé également un certain nombre de questions.

Cet article propose de revenir sur les différentes polémiques et remous que le projet de film d'abord puis le film lui-même ont pu susciter en dressant dans un premier temps une chronologie des étapes importantes du projet. Ensuite, nous reviendrons sur les implications qui ont été les nôtres à Ouvéa, avant de revenir plus longuement sur les arguments pour et contre le film au moment de son écriture puis de la délocalisation du tournage hors d'Ouvéa. Enfin, nous tâcherons d'analyser les principaux échecs et ratés de la production sur le terrain en revenant

5. Plusieurs documentaires ont été consacrés ces dernières années à l'histoire contemporaine d'Ouvéa (Busso, 1998, Faurie, 2005; Drévillon, 2008; Lallaoui, 2008)

6. On pense en particulier aux différents chantiers d'Ouvéa qui mettent des années à aboutir (construction d'une école de voile, réfection de la chefferie de Fajawe, construction d'un nouveau collège public à Lekiny...).

7. Mathias Faurie est docteur en géographie, auteur d'une thèse sur les dynamiques de patrimonialisation en cours à Ouvéa. Mélissa Nayral est doctorante en anthropologie et réalise une thèse sur la vie politique à Ouvéa. 
notamment sur la question de la mémoire et des modalités de sa transmission.

\section{Chronologie du projet et genèse du film}

Après la lecture du rapport de la Ligue des Droits de l'Homme sur « l'affaire de la grotte " (Sanguinetti, 1989) et sur les conseils d'un proche, Olivier Rousset, ayant séjourné à Ouvéa dans sa jeunesse ${ }^{8}$, Mathieu Kassovitz décida d'élaborer un projet de film sur «l'affaire d'Ouvéa ». Olivier Rousset fut le coordinateur principal du projet de tournage à Ouvéa et il a joué dans le film le rôle de l'un des membres du GIGN (Groupe d'intervention de la gendarmerie nationale). Tous deux souhaitaient que la majorité des séquences soient tournées à Ouvéa, sur les lieux du drame avec pour acteurs, figurants et techniciens, des habitants de l'île ayant vécu ces événements.

La trame du film se concentre sur la confrontation entre deux individus dont le destin va basculer : Philippe Legorjus, capitaine du GIGN, et Alphonse Dianou, militant indépendantiste kanak, présenté comme le meneur des preneurs d'otages. Lors de leurs négociations, les deux hommes auraient eu de nombreuses discussions dont la portée philosophique est centrale dans le scénario proposé par Mathieu Kassovitz qui voit dans ces moments une rencontre universelle :

« la rencontre du Blanc et du Noir, du militaire et de l'homme libre, la rencontre de tous ces opposés-là. " (Extrait de la réunion ${ }^{9}$ à la grande chefferie de Weneki, 14/04/2009)

Alphonse Dianou et Philippe Legorjus ont été chacun à leur manière des hommes d'action, liés par un drame humain au cour d'une situation de crise. De la même manière que Philippe Legorjus, Mathieu Kassovitz considère qu'une issue non violente à cette prise d'otage semblait possible et que ces deux personnes ont été rattrapées par des enjeux politiques les dépassant qui les menèrent au dénouement d'une extrême vio- lence que l'on connaît. Le site internet officiel du film relaye d'ailleurs ces commentaires :

« À travers des valeurs communes, ils vont tenter de faire triompher le dialogue. Mais en pleine période d'élection présidentielle, lorsque les enjeux sont politiques, l'ordre n'est pas toujours dicté par la morale... » (http://www.lordreetlamorale-lefilm.com/, consulté le $12 / 07 / 2011)$

Arguant du respect de la « coutume ${ }^{10}$ » et des gens d'Ouvéa ayant vécu ces événements très difficiles, Mathieu Kassovitz et Olivier Rousset s'étaient déplacés dans quelques chefferies de l'île de manière plus ou moins formelle en 2001, afin d'exposer l'idée de ce projet de film. Sept ans plus tard et après d'autres repérages succincts, le réalisateur trouvait une maison de production et des financements. Le projet prenait forme au point qu'en 2008 une équipe complète revint à Ouvéa sollicitant à nouveau certaines chefferies, " l'aire coutumière iaai"1" ", le gendarme Samy Ihage (en poste à la gendarmerie de Fajawe en 1988) ainsi que les différentes institutions locales (mairie d'Ouvéa, province des Îles Loyauté, gouvernement de la Nouvelle-Calédonie, province Sud).

À ce moment-là (2008), le conseil de district Nord (officiellement composé de tous les coutumiers du district Nord d'Ouvéa) exigea que les cinéastes consultent les familles des «dixneuf ${ }^{12}$ » et obtiennent leur accord, sans quoi le conseil s'opposerait au projet. Tout au long de l'année 2008, Mathieu Kassovitz et Olivier Rousset se sont ainsi entretenus avec plusieurs membres de chaque famille. Certains étaient plus ou moins réticents au départ, mais l'équipe affirmera ensuite qu'une majorité avait fini par donner son aval, en acceptant que leurs noms soient divulgués si besoin. Comme M. Waneux l'expliquait dans un entretien accordé au quotidien Les Nouvelles calédoniennes (LNC) :

« La production a envoyé Olivier Rousset qui [...] a rencontré tout le monde. [...] On a eu le feu vert de partout, de la mairie, du sénat coutumier, du président du gouvernement, des provinces et surtout des familles

8. Olivier Rousset, comédien, avait en effet parlé de « l'affaire d'Ouvéa » à Mathieu Kassovitz lors du tournage des Rivières pourpres (2000) et avait évoqué auprès de lui la possibilité de réaliser un film sur ce sujet historique.

9. Réunion organisée à la demande de l'équipe de Nord-Ouest Films afin d'obtenir l'autorisation de tournage à Ouvéa.

Des habitants de Teuta, Heo et Weneki avaient invité Mélissa Nayral à y assister et elle a enregistré la totalité des discussions (plus de quatre heures en tout). Toutes les citations qui y font référence en sont donc extraites.

10. La «coutume» est une institution centrale de l'organisation sociale en Mélanésie. Comprenons ici ce qu'en disait Jean-Marie Tjibaou : «Pour nous, le terme générique de coutume, c'est plutôt le droit, notre manière de vivre, l'ensemble des institutions qui nous régissent. » (Tjibaou, 1996 : 202, éd. orig. 1985).

11. L'aire coutumière est une institution créée par les accords de Matignon en 1988 dont le fonctionnement institutionnel est organisé par la loi organique n ${ }^{\circ}$ 99-209 du 19 mars 1999 relative à la Nouvelle-Calédonie. La Nouvelle-Calédonie compte sept aires coutumières (dont celle d'Ouvéa, l'aire iaai) qui, à l'image du sénat coutumier, sont toutes compétentes pour ce qui concerne la culture kanak.

12. «Les dix-neuf » est l'expression utilisée pour désigner les dix-neuf Kanak tués lors de l'opération militaire destinée à libérer les otages de la grotte. 
des victimes, de l'association des prisonniers. » (Mathias Waneux, Un élu des îles explique le départ de Kassovitz, LNC, 27/05/2010)

Forte de l'accord de plus de trois cents personnes, l'équipe du film revint à Ouvéa en 2009 afin d'obtenir l'autorisation finale de tournage du conseil de district du Nord ${ }^{13}$ et des chefferies auprès desquelles elle sollicita l'organisation d'une réunion. Il était prévu de commencer immédiatement le casting et la construction des décors pour pouvoir entamer le tournage le $1^{\text {er }}$ août 2009 , comme annoncé à la production qui, depuis Paris, commençait à s'inquiéter de ce projet de plus en plus coûteux, complexe et étalé dans le temps.

Lors de cette réunion ${ }^{14}$ destinée à obtenir l'aval du conseil du district Nord, Mathieu Kassovitz présenta longuement et minutieusement les objectifs intellectuels qui lui tenaient à cœur dans ce projet. Il affirmait souhaiter que les gens d'Ouvéa, tant oubliés, en particulier par la France, puissent laisser une trace « écrite » dans l'histoire en transmettant leur expérience. Le deuxième objectif, plus politique, était de faire un film le plus juste possible afin que le « sacrifice des dix-neuf » résonne dans le monde et ne soit pas vain. Enfin, Mathieu Kassovitz souhaitait faire grandir la France en lui faisant découvrir le peuple kanak et la légitimité de son combat et contribuer ainsi au processus de décolonisation du pays qui est, selon lui, loin d'être terminé malgré les accords politiques déjà signés. À la manière de ce qu'il avait effectué pour La Haine (1995), il souhaitait par ailleurs valoriser la population en la sollicitant fortement pour la réalisation du projet afin, notamment, de faire comprendre qui sont les gens d'Ouvéa « à ceux qui n'ont pas l'honneur [...] de pouvoir venir jusqu'ici [Ouvéa] le faire par eux-mêmes » (extrait de la réunion du 14/04/2009), la cible ultime du réalisateur étant de rétablir « la vérité » ${ }^{15}$ sur ce qui s'est « vraiment passé » au moment de l'assaut de la grotte et immédiatement après, afin de dénoncer les manipulations politiques à l'œuvre à cette époque. Il convient toutefois de questionner la légitimité de Mathieu Kassovitz à vouloir rétablir « la » vérité sur cette affaire quand on sait les difficultés inhérentes à l'interprétation d'un fait historique en général et alors même que les versions des faits (ou les « vérités ») diffèrent sur certains points au sein même des Kanak d'Ouvéa.

Pendant la réunion du 14 avril 2009, Mathieu Kassovitz défendait vigoureusement son projet et sa légitimité à traiter ce sujet auprès de l'assemblée. Son envie de soutenir les gens d'Ouvéa aux yeux du monde lui en avait manifestement fait oublier leurs normes de politesse... En s'exprimant fermement et à voix haute lors de cette réunion, Mathieu Kassovitz manqua de respect aux « vieux » présents et c'est parce que Mathias Waneux fera, au milieu de la réunion, une coutume de pardon pour l'excuser d'avoir haussé le ton, que les discussions purent reprendre. Mathieu Kassovitz et Olivier Rousset, comme l'ensemble des membres de l'équipe, ne comprirent la subtilité des normes protocolaires kanak et la complexité des implications locales du projet que progressivement et bien souvent à leurs dépens.

À l'issue de ces longues heures de discussions, le conseil de district Nord donna malgré des craintes ouvertement exprimées un accord de principe pour la réalisation du film.

\section{Sur le terrain}

\section{Notre position de chercheurs}

Dans le cadre de nos recherches, nous avions tout deux effectué plusieurs terrains longs à Ouvéa ${ }^{16}$. Ceux-ci s'inséraient en principe au sein d'un quotidien insulaire, rural et isolé. Lors de l'arrivée des cinéastes, notre pratique de l'enquête ethnographique, qui s'adaptait le plus possible au rythme local, mais surtout à la vie de tous les jours à Ouvéa, s'est trouvée confrontée à une " accélération du temps » ainsi qu'à une relative effervescence, toutes deux venues bouleverser, non sans excès, les structures en place et les fragiles équilibres du quotidien. À propos du film, tout le monde étant concerné de près ou de loin par les événements, chacun pouvait ressentir une certaine légitimité à émettre des recommandations et/ou à jouer un rôle sur une partie au moins du projet. À Ouvéa, la récurrence des

13. La grotte dans laquelle les otages étaient cachés se situe dans le nord de l'île et les « dix-neuf»appartenaient pour la majorité à des tribus du Nord de l'île.

14. Le conseil de district du Nord dans sa forme la plus stricte ne compte qu'un certain nombre de notables (hommes) de chaque chefferie. Ce jour-là, plusieurs femmes étaient présentes et plusieurs autres personnes pour certaines chefferies.

15. On notera que dans l'introduction du film le narrateur dit : « Ceci est une histoire vrai ».

16. Mathias Faurie a mené ses recherches de master 1 et de doctorat entre 2004 et 2011, en particulier dans le district Nord pour un total de quinze mois. Mélissa Nayral a mené ses recherches (master, doctorat) entre 2007 et 2011 pour un total de quatorze mois de terrain à Ouvéa (trois dans le district Sud à Lekiny, cinq dans le district Nord, à Heo-Weneki et six dans le district Centre à Hnyimähä-Hwaadrila). 


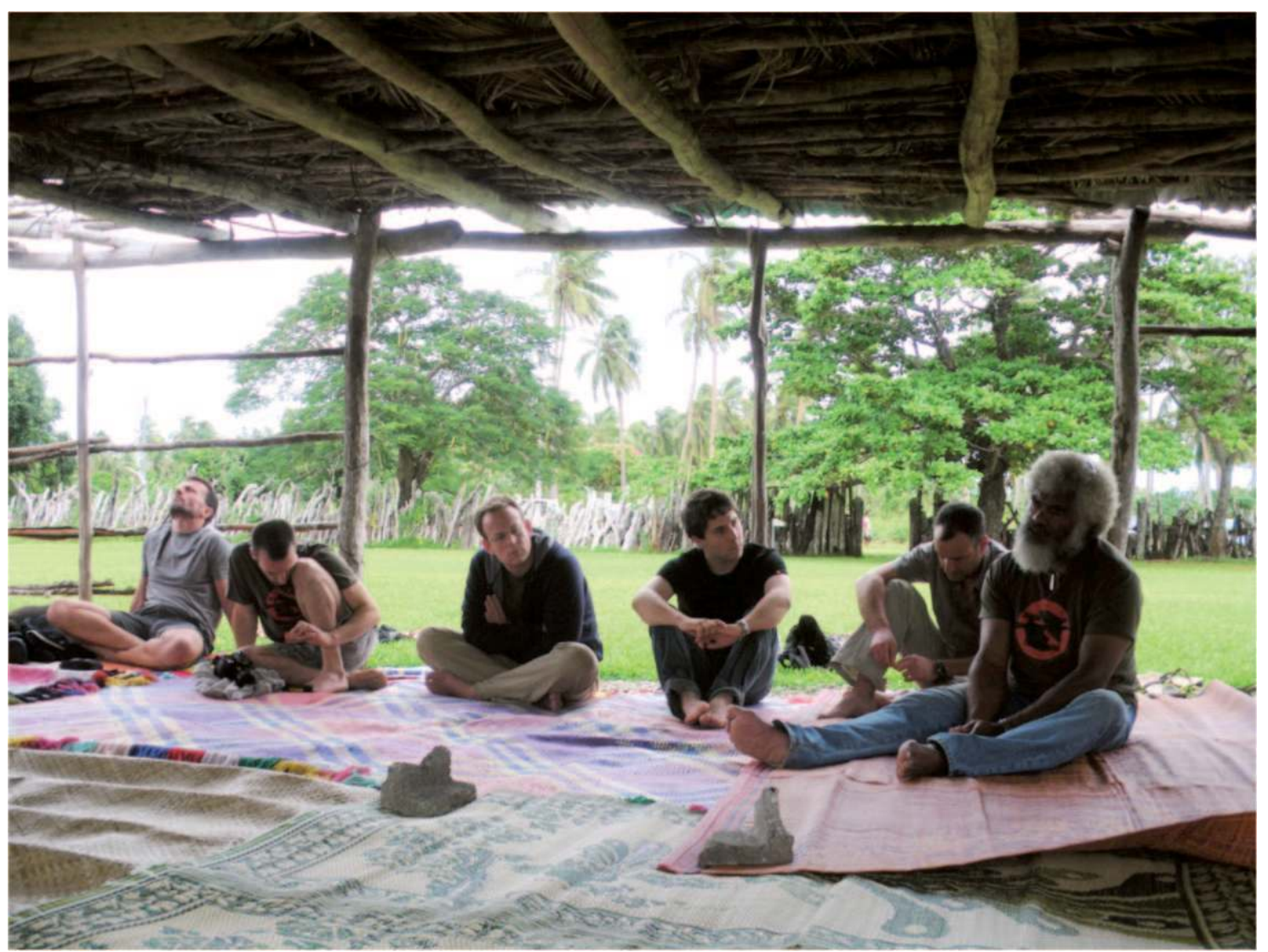

Рното 1. - Réunion du 14 avril 2009 à la grande ghefferie de Weneki (cliché de Mélissa Nayral)

récits concernant les événements dans les conversations quotidiennes est telle qu'il est impossible d'affirmer que les blessures ont été pansées. C'est aussi ce qui explique que le film ait été au centre de beaucoup des conversations que nous avions alors dans le cadre de nos enquêtes.

En intégrant la production Nord-Ouest Films comme deuxième assistant réalisateur (à Paris, dans un premier temps, puis de mars à août 2009, à Ouvéa), Mathias Faurie a directement participé à la préparation du film. Il a assisté le casting, corrigé le scénario, fait des repérages et diverses démarches coutumières et administratives relatives à un tournage initialement prévu sur l'île pour août 200917. Mélissa Nayral était aussi sur le terrain en 2009 et 2010 pour son doctorat en anthropologie portant sur la vie politique à Ouvéa. Elle choisit de continuer ses enquêtes de terrain en portant un regard particulièrement attentif sur les interactions entre l'équipe de tournage et les habitants d'Ouvéa. Elle a plusieurs fois assisté à des négociations et des demandes d'autorisations pour la mise en place du film. En 2009, à Ouvéa, le film n'était encore qu'un projet et beaucoup de nos interlocuteurs naviguaient de l'indifférence à l'engagement partisan, changeant d'avis de manière parfois déconcertante ; certains cependant avaient des avis bien tranchés motivés par des raisons qu'il convient d'exposer.

Les "pour» le film: un film au service de la mémoire collective?

Pour ceux favorables au projet, le film de Mathieu Kassovitz présentait plusieurs pointspositifs. Les représentants de diverses tribus ${ }^{18} \mathrm{du}$ Nord saluaient la démarche de longue haleine et

17. Cette expérience professionnelle s'intégrait dans son doctorat portant sur les recompositions territoriales impliquées par la mise en valeur du patrimoine d'Ouvéa (Faurie, 2011). Son engagement au sein de la production prit fin avec la délocalisation du tournage à Tahiti : le travail mené auprès de la population d'Ouvéa devint alors obsolète car seule une vingtaine d'Ouvéens participèrent finalement au tournage.

18. L'utilisation de ce terme a été initié par l'administration coloniale (sans qu'il n'ait pour autant de pertinence sociologique) et c'est désormais le terme utilité par les Kanak eux-mêmes pour parler de leur village. A ce sujet, voir par exemple Saussol (1985), Leblic (1993), Merle (1995), Lafargue et Nicolau (2003), Naepels (2010), etc. 
l'ample travail de consultation mené par l'équipe auprès des coutumiers d'Ouvéa et des différents protagonistes de l'époque. Comme le plaidait devant eux Mathias Waneux :

«On a rencontré des prisonniers, on a rencontré des mamans, on a rencontre peut-être des frères [...]. C'est pas faute de ne pas avoir sollicité qui que ce soit [...]. Moi je voudrais dire qu'on a rencontré les institutions, on a rencontré euh l'Etat, les responsables de l'Etat, le haussaire de Nouméa et qui eux sont favorables à la démarche. [...]. On a rencontré le sénateur [...] presque toutes les personnes que vous nous avez demandé de rencontrer, on l'a fait. Le chef de Hwadrilla nous a dit, [...] ok. [...]. On a été à la grande chefferie de Fajawe, on a été à l'aire coutumière, on a été voir le président de l'aire. On a vu les gens de l'uc, on a vu quelques personnes du PALIKA [...]. On a été à Lifou pour voir le gendarme Samy. [...]. » (Extrait de la réunion à la grande chefferie de Weneki, 14/04/2009)

Le discours de Mathieu Kassovitz, présentant son film comme engagé pour la cause kanak mais non partisan, devait donner au projet une tonalité honnête et sans compromissions, ni envers la lutte pour l'émancipation ni en terme de réconciliation.

En plus d'être une fiction, ce film, qui se fonde en partie sur des récits de témoins et de protagonistes d'alors, pouvait constituer une forme de «témoignage historique» inédit qui, d'une manière inévitablement romancée, formerait un recueil de données ${ }^{19}$ supplémentaire et original sur cette période trouble. Effectivement, une grande partie des protagonistes (Kanak ou militaires) est encore en vie et ils devaient être impliqués tant dans la construction du scénario que dans le tournage. En voulant devenir un moyen de matérialiser en partie cette mémoire, le film pouvait la rendre exportable: on pouvait par exemple imaginer qu'il puisse être diffusé dans les lycées de Nouvelle-Calédonie ou même de métropole en tant que témoignage historique des « événements ». Le film, tel que présenté à cette étape du projet, possédait en effet une portée géopolitique incontestable : Mathieu Kassovitz souhaitait faire connaître l'histoire calédonienne en France et à l'étranger, mais aussi apporter la lumière sur la lutte kanak et la répression coloniale. Un grand projet comme le sien pouvait peut-être ouvrir les portes d'Ouvéa, en étant un témoignage pour l'humanité d'une partie de l'histoire franco-kanak. Pour certains militants indépendantistes, ce support était ainsi une formidable et inédite occasion de faire entendre la revendication kanak au-delà de l'archipel.

Plusieurs de nos interlocuteurs affirmaient qu'en plus d'être un moyen de panser les plaies de l'histoire, ce film était aussi une occasion unique de témoigner et de transmettre aux générations qui n'avaient pas connu « les événements ». Pour Aïzik Wéa de Goosana :

« Le film est important car les vieux ne seront plus toujours là pour raconter aux jeunes d'ici et surtout de Nouméa l'histoire telle qu'elle a été vécue ici.» (Extrait d'entretien Faurie, 2009)

Ceux qui étaient présents lors de cette crise devaient pouvoir, par l'intermédiaire de ce projet, faire entendre leur voix et décrire les choses telles qu'ils les avaient vécues. Si elle relevait le défi de réaliser ce film, la population d'Ouvéa, souvent marginalisée depuis le double assassinat de Jean-Marie Tjibaou et Yeiwéné Yeiwéné20, bénéficierait alors d'une toute autre image publique, favorable à long terme, notamment en terme de réconciliation mais aussi pour le tourisme. Pour Xavier Tangopi de Takedji :

« Il est avant tout central que l'on reparle d'Ouvéa qui a été plongé dans l'ombre depuis 1988-1989. » (Extrait d'entretien Faurie, juin 2009)

En outre, une participation active de la population locale devait permettre, en particulier aux plus jeunes, d'avoir des perspectives d'emploi en plus d'apaiser un peu leur souffrance en la partageant. Enfin, certains y voyaient aussi une contribution vers l'émancipation politique de la Nouvelle-Calédonie. Pour Marcel Wadjeno, le film devait pouvoir aider :

« à ouvrir les yeux des Français sur la légitimité de la lutte kanak et peser sur le débat qui s'ouvrira dès 2014. » (Extrait d'entretien Faurie, juin 2009)

Tous ces arguments sont ceux qui ont conduit le conseil de district Nord d'Ouvéa à ne pas s'opposer à la mise en place de ce projet. Précisons que, dans ce cas, « ne pas s'opposer » n'est pas un synonyme parfait «d'accepter». La nuance se fonde là encore sur des logiques discursives complexes relatives aux discours publics

19. Dans cette « société de la parole », le support audiovisuel connaît un engouement grandissant.

20. L'assassinat des deux leaders politiques a eu lieu le 4 mai 1989 à Hwaadrilla pendant la cérémonie de la levée des deuils des « dix-neuf» tués lors de l'assaut de la grotte un an plus tôt. Le meurtrier, Djubelly Wéa, était un pasteur de Goosana, militant indépendantiste radical initialement affilié au PALIKA. Considéré comme un leader local, il a été pris à partie par l'armée alors qu'il était souffrant. Il a été incarcéré en France en mai 1988 alors qu'il n'avait pas directement participé à la prise d'otages, puis a été libéré en juillet. Absent aux négociations de Matignon-Oudinot (26 juin et 19 août 1988), il tira sur J.-M. Tjibaou et Yeiwéné Yeiwéné le 4 mai 1989, avant d'être abattu à son tour par un gendarme kanak qui assurait la sécurité des leaders. 
kanak, que les cinéastes n'ont pas non plus sai$\operatorname{sies}^{21}$. C'est certainement ce qui a fait croire à Mathieu Kassovitz à une adhésion totale au film, alors qu'elle n'était que partielle, malgré les avertissements et conseils répétés à cet égard ${ }^{22}$.

\section{Les « contre » : un film qui ravive des clivages?}

Alors que plusieurs Kanak faisaient part d'un enthousiasme certain pour ce projet, celui-ci suscitait aussi de grandes appréhensions qui, en plus d'être nombreuses, dépassaient pour beaucoup le cadre du film. À Ouvéa, elles concernaient avant tout les effets que le film pourrait avoir sur le climat social de l'île. Certains craignaient qu'il ravive les clivages entre les jeunes Kanak qui n'ont pas connu les événements et la population européenne en général ou les gendarmes en particulier.

D'autres pensaient que ce film risquait en outre de réveiller des tensions internes aux familles. Rappelons que pendant les événements, en particulier en 1988, Ouvéa était nettement divisée entre indépendantistes et loyalistes. Certains Kanak d'Ouvéa avaient alors collaboré avec les forces de l'ordre, en leur donnant par exemple des indications sur le lieu où les otages étaient cachés. Plusieurs familles sont depuis scindées et d'autres, comme à Teuta, ne sont réconciliées ${ }^{23}$ que depuis 2008 .

L'idée du film déchaînait aussi les passions de nombreux habitants sur l'ensemble de la Nouvelle-Calédonie. Dès l'annonce de la venue de Mathieu Kassovitz sur le territoire en marsavril 2009, de nombreux blogueurs calédoniens, redoutant un retour des tensions communautaires, avaient manifesté leurs craintes et leur désaccord envers ce projet ${ }^{24}$. À titre d'exemple, voici ce que nous avons pu lire sur un blog :
«L'ordre et la morale: encore une bonne chiasse politicienne en perspective qui n'apportera rien d'autre que la flatterie de l'ethnocentrisme kanak et creusera un fossé qui le sépare des autres communautés. Bienvenue à toi, Mathieu Kassovitz le donneur de leçon. Pitoyable France coloniale. » (Ikarios, http:// caledosphere.com/, consulté le 4 juin 2009) 25

Le sujet du film suscitait également des prises de positions tranchées de la part des personnalités politiques calédoniennes, largement divisées sur le sujet. Rock Wamytan, élu UC du FLNKS ${ }^{26}$ en province Sud et aujourd'hui président du congrès, s'était opposé publiquement au tournage du film. Il avait argué que tous les habitants d'Ouvéa n'étaient pas favorables au projet et que le scénario était trop centré sur Philippe Legorjus qui demeure, aux yeux de nombreux militants indépendantistes, un «traître ». C'est par exemple le terme utilisé dans un communiqué de presse signé par Benoît Tangopi, le "gardien » de la grotte ${ }^{27}$, pour parler de P. Legorjus :

« Nous ne sommes pas contre le film en tant que tel, mais contre le fait que ce film soit basé sur l'histoire de Legorjus qui nous a trahis. » (Tangopi, 2011)

Il ne faut pas oublier, comme le rappellent les rescapés de la grotte, que Philippe Legorjus a fourni des armes aux gendarmes prisonniers en les cachant sur le substitut du procureur Bianconi (Michalski, 2004), tout en faisant croire aux ravisseurs que la télévision française allait venir recueillir leurs revendications en vue d'une issue pacifique ${ }^{28}$. Enfin, Rock Wamytan conteste le fait que le film critique ouvertement la position du FLNKs lors des événements d'Ouvéa en avançant des reproches, selon lui infondés, envers le parti. Certains militants indépendantistes affirment en effet que, pendant toute la durée de la prise d'otage, les responsables du FLNKS, qui

21. Comme l'affirmaient Bensa et Rivierre en 1982, « Dans une société où aucune position n'est unilatérale ni détenue une fois pour toutes, le statut de chaque groupe s'acquiert provisoirement au terme de diverses stratégies et compétitions qui se résolvent dans l'élaboration d'une identité sociale et politique complexe. » (Bensa et Rivierre, 1982 : 14). Plus récemment et toujours à propos de ces logiques discursives complexes de la parole publique en pays kanak, M. Naepels, après d'autres ayant travaillé sur ces questions là, choisissait de parler des « nouages de la vérité » qu'il est nécessaire de maîtriser pour saisir le sens profond et la portée des discours (Naepels, 1998). Comme partout ailleurs, à Ouvéa, la vérité est liée à celui qui l'exprime. Toutefois, en pays kanak où la parole publique peut être considérée comme un acte politique, il est -peut-être encore plus qu'ailleurs- impératif de la replacer dans son contexte avant de commencer à l'interpréter.

22. Pour le cinéaste et les membres de la production, les conseils d'« universitaire » n'apportaient rien d'autre que la complexification du processus et le ralentissement de la mise en place du tournage.

23. La réconciliation est un processus qui requiert un travail dans le temps, concerne toutes les communautés et se poursuit aujourd'hui. Sur ces sujets, voir notamment Mwà véé $(1999,2005)$.

24. Voir en particulier le blog « Calédosphere » de mars-mai 2009 (http://www.caledosphere.com).

25. Le commentaire a depuis été effacé.

26. UC (Union calédonienne), une des composantes du FLNKS (Front de libération national kanak et socialiste).

27. B. Tangopi, ancien preneur d'otage et ancien prisonnier, est présenté publiquement comme le « gardien de la grotte » de Watetö. C'est à ce titre qu'il a par exemple signé un communiqué de presse à propos du film (Tangopi, 2011) Toutefois, et comme souvent en pays kanak, l'appartenance coutumière de ce lieu symbolique est discutée par les tribus voisines. Les événements de 1988 ont largement contribué à remodeler la sacralité et l'appropriation de cet espace.

28. L'hélicoptère d'Antenne 2 était en réalité un leurre qui permit de couvrir et d'orienter la progression terrestre et de déclencher l'assaut en faisant diversion. 
avaient initialement appelé à faire des actions « coup de poing », auraient failli à leurs responsabilités, se rendant volontairement injoignables car étant embarrassés par la situation (à ce sujet, voir notamment Drevillon, 2008. Cette version de l'abandon hiérarchique a également été retenue par Mathieu Kassovitz). En dernier lieu, Rock Wamytan a dénoncé la "manipulation de l'histoire» faite par Mathieu Kassovitz (Nouvelle-Calédonie 1 ${ }^{\text {ere }}, 15 / 11 / 11$ ).

Le contenu du scénario même, en particulier le fait que Mathieu Kassovitz ait choisi d'articuler son film autour de l'expérience du capitaine Legorjus, pose donc un certain nombre de problèmes. Lors de sa présentation au conseil de district Nord d'Ouvéa, Mathieu Kassovitz avait longuement insisté sur le fait que n'étant pas Kanak lui-même, il ne souhaitait pas faire le film à travers les yeux d'un Kanak. C'est pourquoi il avait opté pour le point de vue de Philippe Legorjus (dont il assume le rôle à l'écran), se basant en partie sur son livre témoignage intitulé La morale et l'action ${ }^{29}$ (Legorjus, 1990). C'est d'ailleurs ce qui explique le titre définitif du film $^{30}$, L'Ordre et la morale. Le choix de Philippe Legorjus comme personnage principal du côté français peut être justifié par son omniprésence dans l'action, de Paris à Nouméa puis à Ouvéa (et même à Lifou) puisqu'il s'était beaucoup déplacé pendant ces dix jours afin de rencontrer tous les interlocuteurs.

En termes cinématographiques, cet homme relie les séquences et permet une forme d'incarnation du spectateur étranger à Ouvéa. Mathieu Kassovitz a séjourné chez Philippe Legorjus pour s'imprégner du personnage et en savoir plus que ce qu'il a bien voulu écrire ${ }^{31}$. Il a également interrogé plusieurs militaires qui avaient participé à l'assaut ou à son organisation (tous n'ont pas répondu), afin de recouper ses propos avec des témoignages directs des rescapés de la grotte d'Ouvéa.

Précisons toutefois que les discussions entre Philippe Legorjus et Alphonse Dianou n'ont pas pu être racontées par A. Dianou qui n'a pas survécu à l'assaut de la grotte puisqu'il est mort dans des conditions douteuses lors de son éva- cuation vers le dispensaire de Hulup (Sanguinetti, 1989: 46). Philippe Legorjus lui-même n'en fait que de rares mentions dans son livre, une dizaine de lignes en tout :

« Je profite du climat de décrispation pour entamer une discussion avec Alphonse. Le courant commence à passer entre nous et j'essaye de le tester sur la réalité de son vouloir. Je veux savoir jusqu'où il peut aller, jusqu'où il veut aller et ce qui peut l'arrêter. » (Legorjus, $1991: 227$ )

« J'y passe le reste de la journée en longues discussions avec Dianou et avec d'autres membres du commando. » (Legorjus, 1991 : 233)

«Je découvre depuis une semaine un chemin de traditions et de culture mêlé à des projets politiques utopiques. » (Legorjus, 1991 : 256)

Bien qu'élaborées à l'aune de témoignages de protagonistes de l'époque, ces discussions centrales dans le scénario ont aussi été l'espace privilégié des choix artistiques du réalisateur. Comme il l'a précisé au moment des démarches effectuées à Ouvéa :

« La vraie personne qu'on va voir dans le film, le vrai kanak qui va représenter ce que, plus que, le vrai Alphonse Dianou, ça va être la personne qui va jouer le rôle de Alphonse. Parce que Alphonse, les discussions qu'il a eu avec Legorjus à l'intérieur de la grotte, et qui sont des discussions philosophiques, je n'ai que la version de Legorjus. Mais moi, j'imagine aussi ce qui s'est dit quand je parle avec vous, quand on parle de la nature, quand on parle de la vie, et c'est ça que j'ai envie de mettre dans le film. Et ça va être le seul kanak qu'on va vraiment entendre s'exprimer au nom du peuple. Et ça, ça va être mon point de vue artistique et ma rencontre avec vous. [...]. » (Extrait de la réunion à la grande chefferie de Weneki, 14/04/2009)

Ce paradoxe entre liberté artistique et restitution des faits nous conduit à considérer une des réalités fonctionnelles du projet cinématographique : pour être accessible au grand public et susciter un intérêt pour chacune des séquences, l'histoire doit être scénarisée et romancée, même dans un film qui prétend rétablir la vérité. Ce qui semble nécessaire pour les uns devient ici choquant pour les autres. À la lecture du scénario qui a considérablement évolué entre

29. Plusieurs réalisateurs avaient déjà contacté Philippe Legorjus, mais celui-ci a toujours refusé jusqu'à la proposition de Mathieu Kassovitz. Pour P. Legorjus (Ouest France, 22/10/2010) : "Humainement, nous avons rapidement accroché. Puis nous avons eu un vif débat intellectuel sur sa perception de l'affaire. Il était rentré de Nouvelle-Calédonie avec une vision clairement pro-kanak. Je lui ai fait comprendre que tout n'était pas si simple". Le producteur du film confirme, en laissant planer un mystère : "Nous fabriquons un film politique, engagé qui va dire quelque chose de profond". P. Legorjus fait le même pari : "Il faut regarder en face les moments compliqués de notre histoire. On a encore du mal avec l'Algérie. Le film de Mathieu nous permettra peut-être d'avancer plus vite avec la Nouvelle-Calédonie". ».

30. Le film s'est un temps appelé Rébellion et c'est d'ailleurs resté son titre à l'international.

31. Philippe Legorjus a depuis publié un nouvel ouvrage, Ouvéa, La république et la morale. La préface intitulée « Un héros » est signée de Mathieu Kassovitz (Legorjus, 2011). 
2009 et $2010^{32}$ puisqu'il y a eu pas moins de vingt-et-une versions, beaucoup d'habitants d'Ouvéa qui ont vécu de près le drame et $\mathrm{y}$ ont perdu certains des leurs, commencent à se sentir trahis : ils ne peuvent s'approprier le film et y traduire leur propre version de l'histoire, celle-ci étant abordée à partir du point de vue du capitaine Legorjus et romancée selon les choix artistiques du réalisateur et les besoins de l'industrie cinématographique. Pour Joël, ancien preneur d'otages :

« Le scénario ne parle que du point de vue de Legorjus, mais n'aborde pas suffisamment le contexte des événements et de la lutte kanak des années 1980. Ce n'est pas l'histoire que les anciens de la grotte veulent raconter aux Kanak et aux Français, ce n'est pas non plus le film des habitants d'Ouvéa. Ce film est comme une arme qui peut se retourner contre nous. » (Extrait d'entretien Faurie, 2009)

Le projet semblait en 2009 échapper à une partie des habitants d'Ouvéa qui n'arrivait pas non plus à accepter les séquences comiques et celles inventées pour enrichir le film ${ }^{33}$, ni tolérer certains des raccourcis permettant de faire tenir dix jours de drames en cent vingt minutes. Il en est de même pour les simplifications de l'histoire et les anachronismes ${ }^{34}$ visant à permettre au spectateur d'appréhender une réalité complexe et imbriquée et de se faire une idée globale sur la revendication nationaliste kanak et la période des événements en Nouvelle-Calédonie.

Précisons par ailleurs qu'outre les difficultés de construction du scénario, l'équipe de Mathieu Kassovitz a longtemps négligé le travail de consultation des familles d'Ouvéa qui résident à l'extérieur de l'atoll, en particulier dans le « Grand Nouméa » où les Ouvéens sont aussi nombreux que sur leur territoire d'origine. Beaucoup d'entre elles ont aussi vécu les événements en 1988 et certains notables de l'île voulaient légitimement être consultés pour ce projet (choix des acteurs, modifications du scénario). En mars-avril 2009, Mathieu Kassovitz semblait ainsi vouloir produire le film avec les habitants d'Ouvéa tout en s'affranchissant d'un contexte calédonien complexe et imbriqué : les autorisations coutumières et l'aval de nombreux protagonistes de l'époque, extérieurs à l'atoll, ayant jusqu'alors été négligés.

L'ensemble des arguments en défaveur du film avancés en 2009 n'ont pas cessé d'accompagner le projet et ils laissaient finalement présager la suite : trois équipes et directeurs de productions se succédèrent et, de déboires en catastrophes, durent faire face à de nombreuses difficultés. Sur le terrain, rien ne se passait comme prévu alors que le contexte paraissait plutôt favorable étant donné que les cinéastes avaient rencontré plus de trois cents personnes et que - paradoxalement ? - les autorisations formelles avaient été données, l'ensemble des responsables de toutes les familles des victimes, de toutes les tribus, de toutes les associations liées aux événements ainsi que la majorité des institutions avaient déclaré soutenir le projet.

\section{Des difficultés et des ratés qui mèneront à l'annu- lation du tournage à Ouvéa}

\section{Un casting difficile}

Une fois l'accord oral du conseil de district Nord d'Ouvéa obtenu au mois d'avril 2009, tous les membres de l'équipe, sauf le coordinateur local, Olivier Rousset, repartirent en métropole dans les jours qui suivirent. La semaine suivante, deux hommes chargés des castings arrivèrent de Paris. Leur objectif, trouver un maximum de gens (environ 250 personnes) en un minimum de temps (un mois). Ils affirmaient avoir une capacité de rendement s'élevant à environ quatre-vingts essais par jour. En un mois à Ouvéa, ils ne parvinrent à en faire qu'une cinquantaine, déplorant un réel manque de coopération de la population (difficultés à prendre des rendez-vous, peu d'indications pour trouver les maisons, annulations récurrentes, inefficacité des arguments du casting en terme de rémunération...).

32. Le scénario, réclamé avec insistance depuis le début de ce projet (2007) par les autorités coutumières, les familles des « dix-neuf » et l'association des anciens prisonniers d'Ouvéa, a trop tardé à être fourni par la production aux habitants. Des copies circuleront au compte goutte, à partir de fin 2009, malgré l'édition d'une quinzaine de versions depuis 2007, que l'équipe du film n'avait pas été autorisée à divulguer aux habitants. Ceci a contribué à alimenter les critiques relatives au manque de transparence du projet de Mathieu Kassovitz, lui-même rarement présent sur le terrain et pilotant le projet depuis Paris ou Nouméa. Un important travail de remaniement du scénario, séquences par séquences, a néanmoins eu lieu au fil des mois prenant en compte un certain nombre de remarques de certains protagonistes de l'époque, puisque c'est la version 21 qui a été tournée.

33. Pour ne citer qu'un exemple parmi d'autres, une scène où le capitaine Legorjus fume "le calumet de la paix » avec A. Dianou devant la grotte a été retirée du scénario à la demande de la famille de ce dernier car celui-ci était non-fumeur.

34. On pense à titre d'exemple au tee-shirt « fête du Walei » que porte Alphonse Dianou pendant tout le film. La première édition de la « fête du walei » n’a eu lieu qu'en juillet 2009. 


\section{CASTING FILM}

Bonjour à la population d'Ouvéa,

Grâce à l'accord du conseil de l'aire coutumière d'Ouvéa celui des grandes Chefferies du Nord et des personnes liées aux évènements de $\mathbf{8 8}$, nous préparons actuellement un FILM de Cinéma réalisé par Mathieu KASSOVITZ qui racontera l'histoire des 19 tombés à la grotte.

Le film se tournera à Ouvéa à partir du mois d'août 2009.

Nous sommes Marco et Hervé et notre travail consiste à faire le Casting, c'est à dire à trouver les ACTEURS qui joueront dans ce film.

Nous cherchons à rencontrer pour ce Casting des Hommes entre 20 et 50 ans.

Pour ceux qui seront choisis pour des rôles, il s'agira d'un travail rémunéré.

Nous avons besoin de rencontrer un MAXIMUM de monde, car nous chercherons également des gens pour la figuration.

Tous ceux qui sont intéressés pour nous rencontrer, ou ceux qui souhaitent plus de renseignements peuvent nous joindre aux numéros suivants :

HERVE : 980231 ou MARCO : 980251

Ce Film ne peut exister qu'avec votre participation à tous. Oleti.

Doc. 1. - Annonce A4 du casting apposée dans les commerces et lieux publics d'Ouvéa. Certaines affiches ne resteront en place que quelques heures. Quelques jours après leur affichage et devant l'absence d'appels, la production prenait la décision d'organiser elle-même des réunions en tribu, afin d'inciter les habitants à participer aux essais filmés (document collecté en mai 2009 à Ouvéa)

Le problème central était que, parmi ces cinquante essais, il n'y avait personne pour jouer le rôle principal, celui d'Alphonse Dianou. Aujourd'hui, Alphonse Dianou aurait environ 50 ans et, pour les gens d'Ouvéa, loin d'être un personnage imaginaire, Alphonse reste avant un oncle, un cousin, un père ou un frère et personne n'était disposé à endosser une telle responsabilité. Par ailleurs, Alphonse étant un personnage central dans le film, cet acteur charismatique et doué en improvisation devait aussi posséder une réelle maîtrise du français afin de pouvoir mémoriser une grande quantité de dialogues.

$\mathrm{Au}$ bout de plusieurs mois, le casting n'était pas terminé et certains Kanak, qui avaient pourtant fait des essais filmés, ne souhaitaient plus participer au film. D'août 2009, le tournage fût ajourné au printemps 2010 et à Ouvéa les tensions augmentèrent.

\section{Un tournage reporté, annulé puis délocalisé}

$\mathrm{Au}$ fil des mois, les divisions et polémiques devenant trop importantes, la sécurité et la faisabilité du tournage commençaient à être sérieusement mises en doute. Certains opposants au film rédigèrent une pétition alors que d'autres faisaient tout pour annuler le tournage, allant jusqu'à menacer le réalisateur (entretien avec Olivier Rousset, Faurie, 2009). Le risque que le projet soit «bloqué » en plein tournage, voire que certaines menaces physiques soient mises à exécution, était pris très au sérieux par la production qui, ayant déjà investi beaucoup d'argent, ne pouvait se permettre davantage de déconvenues ou de reports supplémentaires. Plusieurs habitants d'Ouvéa avaient fait part à la production de leurs inquiétudes dans le cas d'un tournage sur place et d'un risque de conflits entre partisans et opposants, qui aurait certainement conduit les représentants coutumiers à retirer leurs autorisations. Devant l'ensemble de ces risques potentiels, les interlocuteurs privilégiés de la production à Ouvéa demandèrent finalement à l'équipe de quitter l'île et de trouver un autre lieu de tournage. La production décida alors de le délocaliser vers Anna en Polynésie française $^{35}$. Pour Mathias Waneux, coordinateur local du film :

« Il était prévu de tourner le film ailleurs, en conservant le même esprit, si les conditions de sécurité des acteurs n'étaient pas garanties. » (LNC, 27/05/2010)

Cette délocalisation sonna le glas d'un projet qui se voulait être réalisé avec les habitants d'Ouvéa et rejoué par eux sur les lieux mêmes du drame. Le film ne pourrait définitivement être qu'une fiction "classique » où les décors et la mise en scène primeraient sur la reconstitution populaire in situ. Le 18 août 2010, La Dépêche de Tahiti titrait «Kassovitz dérange en Kanakie [sic !] » et le 24 août, Les Nouvelles calédoniennes écrivaient « Kassovitz divise Ouvéa ».

\section{Des difficultés logistiques et budgétaires}

On sait aussi que l'armée française n'avait pas voulu apporter d'aide logistique au film, mises à part les nécessaires autorisations de survols et de navigation. Tous les hélicoptères, avions, chars et camions militaires allaient devoir être recréés par l'équipe du film (d'abord en Nouvelle-Calédonie et de nouveau à Tahiti). Par ailleurs, plusieurs retards avaient déjà entraîné une perte de fonds importante, en particulier, en mars-avril 2009, lorsque les cinéastes utilisèrent fréquemment des 


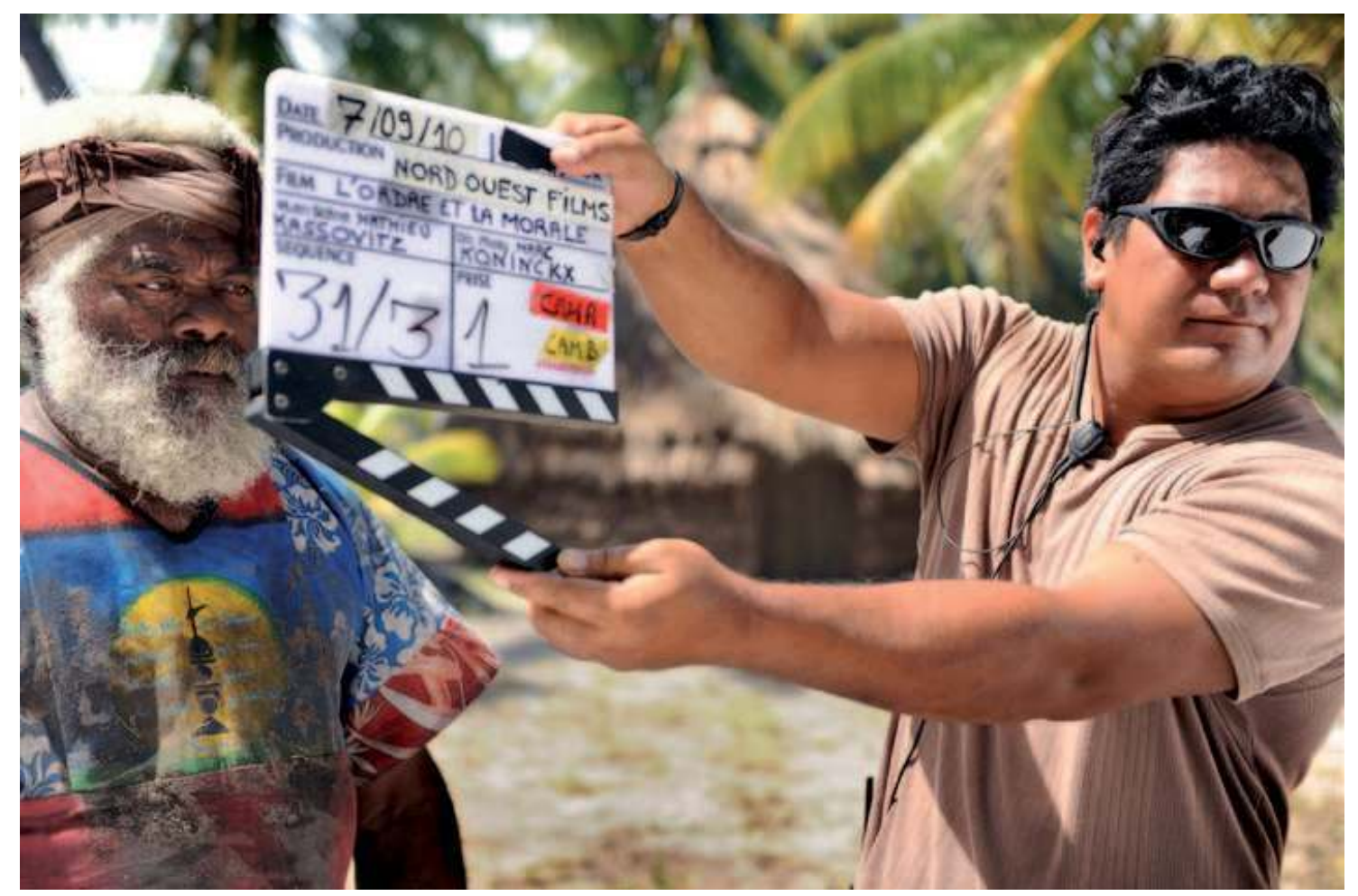

Рното 2. - Extrait du tournage en Polynésie française (C Nord-Ouest Films, 2011)

avions privés pour se déplacer entre Nouméa et Ouvéa suite aux grèves de transports qui paralysaient la Nouvelle-Calédonie. Si la part d'obstacles matériels et financiers n'est pas à négliger ${ }^{36}$, il semble néanmoins qu'elle ne puisse suffire à expliquer les motifs de cette situation clivée qui a notamment conduit à la délocalisation du tournage à Tahiti, lui aussi critiqué par des habitants d'Ouvéa qui, forcément moins nombreux que chez eux, avaient l'impression que le sujet allait leur échapper définitivement. Déjà sensible, la question de la mémoire et de la légitimité de sa transmission devenait dans le cadre d'un tournage extérieur à l'île véritablement problématique. La vingtaine de Kanak qui allaient participer au projet avait-elle une légitimité suffisante pour raconter ce drame?

\section{La mémoire et sa transmission, qui est légitime?}

Il faut comprendre que derrière l'idée de l'implication de la population locale dans le projet, se posait la question de la mémoire collective dans un premier temps et des modalités de sa transmission dans un second. En effet, beaucoup de « gens d'Iaai » (l'autre nom d'Ouvéa) pensent que leur mémoire n'appartient qu'aux Kanak de l'atoll et tout le monde ne souhaite pas qu'elle soit transmise par quelqu'un de l'extérieur, français de surcroît comme l'indique le témoignage d'Adèle, femme de Heo :

« Non, mais c'est quand même son armée qui a tué nos frères là ! Lui, il croit que il peut venir et raconter ça ici chez nous à Ouvéa ! Ouvéa, c'est chez nous, les gens d'Ouvéa, c'est pas les Français qui vont décider de... comment on raconte! Puis nous on a vécu de près les événements... ! Mais c'est sûr que nous, on [ne] fera pas le film là ! Je sais pas c'est qui les gens qui ont dit oui, mais ils sont bêtes ces gens-là, [...] mais nous on pleure des fois. » (Extrait d'entretien Nayral, mars 2009)

Cette situation est caractéristique des difficultés à prendre en charge l'histoire locale et sa transmission aux générations futures ${ }^{37}$. Là encore, sur des points précis tels que la gestion des échéances du calendrier et la pratique du consensus, on ne peut que constater les difficul-

36. Pendant la promotion du film en Métropole, Mathieu Kassovitz répètera d'ailleurs à plusieurs reprises que ce projet a ruiné sa maison de production personnelle et qu'il a régulièrement songé à l'abandonner en raison notamment de son coût qui semblait augmenter sans fin.

37. NDLR. - Problèmes des plus classiques en anthropologie sociale, notamment à propos de la transmission des histoires de clans comme l'a constaté en autres I. Leblic à Ponérihouen (voir ci-dessus et Leblic, 1993) ou M. Naepels à Houaïlou (Naepels, 1998). 
tés de cohabitation entre des façons de faire «plus européennes » et d'autres plus «kanak» relatives dans le cas du film, à leur modalités respectives d'appropriation et de diffusion de l'histoire collective. En pays kanak, la transmission de la mémoire doit se faire de manière intergénérationnelle, à la demande de celui qui voudrait savoir ou selon la volonté de celui qui transmet, mais cela n'est possible qu'à condition que cette transmission soit autorisée par la personne considérée comme détentrice de ce savoir. Certains discours, certains mythes ou contes par exemple, n'appartiennent qu'à certains clans ou lignées (voir ce qu'en dit Jean-Marie Tjibaou dans ses textes republiés en 1996).

Par ailleurs, le fait que des personnes se soient exprimées au nom d'un groupe (clan, tribu, etc.) en donnant un avis favorable au projet de film n'implique pas ici que chaque personne dudit clan par exemple ait accepté de s'investir dans ce projet à titre personnel. Précisons à cet effet que les mots clans, familles, tribus et chefferies sont tous polysémiques et que les frontières des groupes qu'ils désignent peuvent fluctuer en fonction des contextes et qu'à l'intérieur de ces mêmes groupes, les relations d'autorité ne sont pas équivoques. Comme le précise par exemple Isabelle Leblic :

« les rangs sont relatifs, réversibles, selon l'ancienneté dont on peut se prévaloir [...]. » (Leblic, $1993: 84$ )

Ce sont précisément ces segmentations qui ont pu faire croire à Mathieu Kassovitz que l'adhésion était totale alors qu'elle n'était que partielle, ce qui était pourtant évident pour nous, chercheurs sur le terrain. En contexte kanak, si la parole est essentielle, sa portée ne peut être saisie qu'une fois interprétée dans l'espace et le temps et il est difficile d'obtenir un accord ferme et engageant sur des sujets aussi sensibles au sein du monde kanak que le sont ceux qui concernent les événements ${ }^{38}$.
Des enjeux plus pragmatiques, un projet récupéré?

Il est certain aussi qu'à l'échelle d'Ouvéa, d'autres enjeux politiques plus pragmatiques sont à prendre en considération. La commune voyait par exemple dans ce projet l'opportunité de faire raccorder, la mairie dans un premier temps et toute l'île ensuite, au réseau ADSL récemment installé sur la Grande-Terre et dans les autres îles Loyauté, l'engagement pris auprès de l'équipe de Mathieu Kassovitz de pouvoir disposer d'une connexion internet puissante et efficace pendant le tournage servant alors d'argument solide auprès de l'entreprise des télécoms, sous couvert du développement économique que ce film était censé engendrer ${ }^{39}$ (entretien avec le secrétaire général de la mairie d'Ouvéa, Nayral, 2009).

La province des Îles Loyauté elle aussi a bien eu connaissance du projet. Il s'avère que le directeur de l'antenne d'Ouvéa ${ }^{40}$, Mathias Waneux, est en partie à l'origine du film avec Mathieu Kassovitz qu'il connaît personnellement depuis $2001^{41}$. C'est lui qui a guidé les cinéastes pour la plupart des négociations coutumières. Il accueillait Mathieu Kassovitz et Olivier Rousset chez lui, les orientait régulièrement vers divers interlocuteurs et les a accompagnés à la réunion au conseil du district Nord durant laquelle il avait affirmé qu'ils "étaient un peu ses enfants ${ }^{42}$ » avant de s'excuser pour les mots de Mathieu Kassovitz ${ }^{43}$.

M. Waneux, fraichement élu à la province ${ }^{44}$, souhaitait aussi tirer parti de la réussite de cette entreprise en se montrant sous le jour d'un homme responsable, sachant innover pour faire avancer son île et la lutte pour l'indépendance, tout en ne négligeant pas la coutume puisqu'il se présentait comme le responsable des démarches coutumières des cinéastes (LNC, 27/05/2010). Il revendiquait également l'initiative d'une telle

38. NDLR. - On pourrait élargir le propos et mentionner que c'est souvent le cas, quel que soit le sujet, et un oui dit à un interlocuteur, qui plus est, Blanc, est souvent un non. Ou bien on obtient en réponse à nos questions ce que nos interlocuteurs pensent que l'on attend d'eux (à ce propos, Leblic, 1993).

39. Les voyages « à vide » des barges reliant Ouvéa à la Grande-Terre utilisées par la production pour la logistique du film devaient permettre d'effectuer des transports de matériels publics et de rapatrier des déchets encombrants sur l'atoll (carcasses de camions, etc.) (Guinal Riou, régisseur général de Nord-Ouest Production, com. pers. Faurie, 2009).

40. La province des Îles Loyauté est basée sur l'île de Lifou mais il existe une "annexe » à Maré et une autre à Ouvéa.

41. Olivier Rousset avait séjourné chez Mathias Waneux dans sa jeunesse. Ce dernier l'avait à nouveau accueilli en 2001 lors de sa venue avec Mathieu Kassovitz.

42. « Voilà, Mathieu et les garçons quand ils viennent ici à la maison, c'est un peu mes enfants. C'est un peu mes enfants donc c'est pour ça, je vais prendre un peu la parole. » (Extrait de la réunion à la grande chefferie de Weneki, 14/04/2009).

43. Voir ci-dessus la chronologie du projet.

44. Mathias Waneux a été élu en mai 2009 sur la liste de l'Union calédonienne (UC), un des partis indépendantistes membres du FLNKs (auquel appartenait aussi Jean-Marie Tjibaou, Yeiwéné Yeiwéné et Alphonse Dianou). Il a été un membre actif des initiatives de réconciliation autour des événements d'Ouvéa (voir par exemple Mwa véé, 1999, 2005). 
démarche de consultation censée faire de ce film «le premier film au monde pour lequel un peuple a été consulté » (extrait d'entretien, Nayral, avril 2009). Par l'intermédiaire de ce projet, le directeur de l'antenne provinciale gagnait du prestige au-delà du territoire en devenant, au plan international, l'interlocuteur kanak privilégié des cinéastes. Si cette personnalité politique était effectivement un acteur clé du projet de film, elle n'était pas pour autant le "grand chef d'Ouvéa » ou le «leader de la prise d'otages » (La voix du Nord, 08/11/2011), qu'elle est devenue par ricochet médiatique. Les projets d'envergure sont un espace tout à fait approprié à ces jeux de pouvoirs et de statuts et servent d'arènes en pays kanak.

Les lacunes de l'équipe du film sur la connaissance du terrain et le refus de considérer les conseils de chercheurs documentés sur Ouvéa et son fonctionnement social et coutumier ${ }^{45}$ ont contribuées à faciliter une certaine instrumentalisation du projet par des personnalités politiques locales, dont la légitimité à porter la parole en tant que «dépositaires de l'histoire» a été contestée localement. Ainsi, le projet de film a pu agir comme un révélateur mettant en abîme, à travers la question de la légitimité, les nouveaux jeux de pouvoirs locaux et les tentatives de récupération de l'histoire à des fins tout à fait actuelles. Ces jeux de pouvoirs ont en retour contribué à mettre en péril la faisabilité du tournage avec la population d'Ouvéa.

\section{Légitimité}

À l'image de ce qui existe ailleurs en pays kanak, Ouvéa comporte un système de représentativité par la chefferie dont l'autorité est régulièrement discutée par les clans locaux ${ }^{46}$. Cette fragile légitimité ainsi que celle des représentants institutionnels quels qu'ils soient (aire coutumière, gouvernement, etc.) semblent être l'une des caractéristiques de la microsociété d'Ouvéa, où les divisions internes sont exacerbées. Le fait
qu'Ouvéa soit une petite île qui a subi un violent traumatisme contribue à renforcer ces clivages. De plus, à Ouvéa, l'information circule mal et les familles semblent éviter les sujets épineux tant la cohésion sociale est chose fragile et se cultive au quotidien. Enfin, il semblerait que les mêmes interlocuteurs "privilégiés» soient toujours informés et sollicités afin de donner leur avis et que d'autres personnes soient, plus ou moins volontairement, systématiquement écartées des processus publics.

À propos de la légitimité des différents interlocuteurs locaux du projet, on comprend que, là encore, ceux qui sont les plus légitimes pour les uns ne le sont justement pas pour les autres. Ceci est un fait récurent et concerne l'ensemble des recherches en sciences sociales réalisées en pays kanak. La préparation du film s'est greffée sur des fragmentations qui ont finalement conduit à sa délocalisation. Ces divisions - catholiques/ protestants, loyalistes/indépendantistes, iaai/ fagauvea, etc., -, si elles sont loin de résumer Ouvéa, caractérisent malgré tout l'atoll depuis des décennies et ont toutes été exacerbées au cours des événements. Les clans fagauvea ont payé par le plus grand nombre de morts lors de l'assaut de la grotte et étaient très investis au sein de la lutte indépendantiste bien qu'ils ne soient régulièrement pas considérés comme les « vrais gens d'Iaai ». De leur côté, les Iaai de Goosana, souvent alliés avec les clans de Hwaadrila (d'où est originaire Mathias Waneux), n'ont pas versé un lourd tribut lors de l'opération militaire ${ }^{47}$. En revanche, la grotte se trouvant à proximité de leur village ${ }^{48}$, ils se sont trouvés en première ligne de la confrontation et de la violente occupation de la tribu par l'armée ${ }^{49}$ et certains font partie de ceux qui dénoncent encore des actes de torture (Sanguinetti, 1989; Michalski, 2004).

Les habitants de Goosana, en particulier deux des frères de Djubelly Wea, ont été relativement plus enclins à s'impliquer dans le projet de Mathieu Kassovitz à l'inverse d'autres chefferies du Nord de l'île ${ }^{50}$. L'omniprésence de la « grotte

45. En tant qu'universitaire et deuxième assistant mise en scène, les remarques méthodologiques de Mathias Faurie, pourtant récurrentes, n'étaient pas les bienvenues. Les cinéastes le renvoyaient sans cesse à ses méthodes de chercheur en sciences humaines auxquelles ils tenaient fermement à opposer les leurs. Mélissa Nayral a également tenté, sans plus de succès, de fournir des clés de compréhension de la réalité sociale d'Ouvéa, en particulier à propos des clivages entre gens du Nord et gens du Centre, aux responsables du casting après que le groupe de femmes de la tribu de Heo leur ait réservé un accueil glacial.

46. La plupart des chefferies de la Nouvelle-Calédonie ont été mises en place par l'administration coloniale sans se soucier de suffisamment des diverses légitimités coutumières.

47. Waïna Amossa n'était pas un preneur d'otages mais un « porteur de thé » ravitaillant les otages. Il a été tué à l'issue de l'«opération Victor».

48. La grotte dite de Watetö se trouve entre Goosana et Onhyat. Elle est isolée au cœur de la forêt. Son nom et son appartenance coutumière sont discutés par les clans du Nord : les événements de 1988 et le traitement exogène de l'histoire ont renforcé l'emprise de la tribu de Goosana sur cet espace, cette grotte devenant « la grotte de Goosana ».

49. On sait que pendant cette occupation, les gens de Teuta qui avaient fuit l'arrivée de l'armée dans leur tribu en s'échappant par la forêt, étaient avec ceux de Goosana.

50. La chefferie de Teuta s'était également prononcée en faveur du projet. 
de Goosana » dans les médias et les rétrospectives consacrées à l'affaire d'Ouvéa ont longtemps fait croire au réalisateur et à Olivier Rousset que l'adhésion des notables et la participation des habitants de cette tribu primeraient dans l'acceptation du projet. Certaines tribus, comme Teuta, Heo ou Takedji, critiquent la légitimité des personnes de Goosana à s'exprimer au nom de tous, notamment au moyen de ce film. Des divisions demeurent entre les différentes tribus d'Ouvéa qui, si elles ne sont pas forcément frontales ou toujours exprimées dans la violence, sont suffisamment importantes pour empêcher une réelle coopération ${ }^{51}$ sur des projets d'envergure, comme celui de L'Ordre et la morale.

\section{Conclusion}

Contrairement à ce qui a été largement affirmé par les détracteurs du film, le réalisateur a récolté ses données dans des sources variées (archives de journaux télévisés de l'époque ${ }^{52}$, livres, articles de presse et images connus parlant du drame d'Ouvéa, etc.). Des versions émanant des anciens prisonniers, des familles et témoins, en partie recueillies, ont également été croisées avec les autres données et témoignages. Le film, aidé par des capacités financières hors normes, a finalement réuni une grande quantité de données sur le sujet comme peu de personnes avaient eu les moyens de le faire auparavant. Toutefois, plusieurs écueils sont à relever et les équipes engagées dans le projet témoignent, malgré ce travail de documentation, d'une compréhension trop superficielle du contexte social. Celle-ci nous paraît être responsable, au moins en partie, d'une difficile médiation avec la population et du fait que beaucoup de gens d'Ouvéa n'aient pas voulu prendre part à ce projet. Il était, selon nous, impossible de penser obtenir l'accord de tous sur un tel projet et une des erreurs de M. Kassovitz a sans conteste été de croire l'inverse.

La configuration atypique de cette petite société insulaire isolée et divisée n'a pas suffisamment retenue l'attention des cinéastes qui, malgré de nombreux conseils et avertissements, s'en sont tenus à leurs préjugés et à la parole de leurs interlocuteurs initiaux. On l'a dit, Ouvéa reste marquée par ses clivages et, dans les dis- cours, les catholiques sont souvent opposés aux protestants, les locuteurs du iaai à ceux du fagauvea, les indépendantistes aux loyalistes, les gens du district Nord à ceux du Sud et ceux du Centre à ceux du Nord et du Sud, etc. Cette non prise en compte du contexte dans ses détails est incontestablement à l'origine de nombre de « ratés », de frustrations et de critiques abondantes à l'échelon local. Si l'analyse du film, de la mise en place du projet à sa sortie en salles, peut être riche en enseignements sur les structures et les stratégies sociopolitiques locales, il est clair toutefois que celles-ci n'ont que trop peu retenu l'attention des cinéastes, si tant est qu'elles ne leur aient pas complètement échappé. Le réalisateur et son équipe, aveuglés par leurs certitudes, régulièrement accaparés par des préoccupations pratiques, budgétaires et logistiques, ont réalisé bien trop tard que leur projet s'était retrouvé piégé au sein des jeux de pouvoirs et des tensions que connaît l'île d'Ouvéa depuis 1988-1989 malgré sa récente et fragile réouverture sur le monde extérieur.

À la sortie du film au cinéma, il va sans dire qu'une certaine amertume demeure pour les partisans locaux du tournage à Ouvéa. Par ailleurs, du point de vue économique, la délocalisation du projet en Polynésie française a été une mauvaise affaire pour la Nouvelle-Calédonie ${ }^{53}$ et pour Ouvéa. Mais c'est surtout de l'avenir du territoire et du traitement de son histoire qui ne font en rien consensus, dont il a été question autour de ce projet de cinéma.

En effet, les imperfections et inexactitudes relevées dans le film ne remettent en cause ni la nécessité de parler du sombre passé de la Nouvelle-Calédonie, au sein duquel Ouvéa tient depuis l'affaire de la grotte en 1988 et le double assassinat de Jean-Marie Tjibaou et Yeiwéné Yeiwéné en 1989 une place particulière, ni l'exigence de poser des questions de fond sur la politique française outremer hier et aujourd'hui, la (dé)colonisation en général et le devenir institutionnel de la Nouvelle-Calédonie en particulier. Le film de Mathieu Kassovitz remet en cause à juste titre l'action de l'armée française en 1988 en Nouvelle-Calédonie et pose ainsi des questions politiques cruciales auxquelles il faudra pouvoir répondre ${ }^{54}$. Rappelons que :

51. On pense en particulier aux désaccords sur les propriétés foncières ainsi qu'à la légitimité de la chefferie Imwönë dont dépend la plupart des habitants de Goosana, ainsi qu'à la très vieille « concurrence » entre la chefferie Bazit (ou Bahitr) et Hweneguëi (Guiart, 1992 ; Izoulet, 2005).

52. NDLR. - De nombreuses sources sont ainsi en ligne sur le site de la promotion du film (voir http://www.lordreetlamoralelefilm.com/\#/nav/home, consulté le 14/03/2012).

53. Un million et demi d'euros avaient déjà été dépensés en Nouvelle-Calédonie (hôtel, transports, salaires ; etc.).

54. Comme A. Muckle en fait état : « Les références aux conflits passés (principalement 1878 ou 1984-1988) fournissent une toile de fond importante à de nombreux romans, pièces et nouvelles récents qui constituent la littérature néo-calédonienne. " (Muckle, $2010: 53$ ) ; L'Ordre et la morale ne fait pas exception. 
« la constitution interdisait de se servir de l'armée sur le territoire de la République ${ }^{55}$. Pour la deuxième fois, [...] en Nouvelle-Calédonie, le pouvoir de l'époque, Jacques Chirac ${ }^{56}$, a violé la constitution. » (Michel Rocard, 14/11/11, Europe 1 à 8h15)

Toutefois, il semble que si ce film a permis à certains Kanak de partager une mémoire douloureuse, l'industrie cinématographique n'a pas non plus eu le pouvoir - le dessein ? - d'exercer une thérapie collective efficace, ni pour les Kanak d'Ouvéa, ni pour l'ensemble des habitants de la Nouvelle-Calédonie. Les réactions radicales de certains témoignent des violences morales qui caractérisent le territoire encore aujourd'hui, à l'image de celle D. Hickson, directeur des principales salles de cinéma de Nouméa, qui après avoir acheté l'exclusivité des droits de diffusion, décidait de ne pas le projeter, le jugeant trop polémique avant même de l'avoir visionné. Rassurons les détracteurs du film en rappelant que comme tout document cinématographique, celui-ci demeure le produit de la sensibilité et de l'interprétation d'un cinéaste qui en l'occurrence n'est ni un sociologue ni un historien. En ce sens, si L'Ordre et la morale constitue sans conteste un «document » supplémentaire sur les événements d'Ouvéa, il ne peut pour autant se substituer à l'ensemble des travaux effectués sur le sujet depuis vingt ans, qu'il doit au contraire nous inciter à consulter.

\section{BIBLIOGRAPHIE}

Adck-Centre Tubaou, 1999. Mwà véé 26 : Usooköu, Ouvéa, le temps de la réconciliation.

—, 2005, Mwà véé 46 : Pardon et réconciliation.

Bensa Alban et Jean-Claude RiviERre, 1982. Les chemins de l'alliance, Paris, Selaf., 586 p.

Busso Frédy et Nathalie BARBE, 1998. Ouvéa, l'âme kanak, 54 min, Paris, Images Plus-Sombrero Productions.

Drévillon Élisabeth, 2008. Grotte d'Ouvéa. Autopsie d'un massacre, 64min, Galaxie presse.

FAURIE Mathias, 2005. Ouvéa, le développement pour l'indépendance?, 35min, autoproduction.

Guiart Jean, 1992 ( $1^{\text {re }}$ éd. 1963). La chefferie en Mélanésie, $2^{\mathrm{e}}$ éd. remaniée et augmentée, Paris, Institut d'ethnologie, $464 \mathrm{p}$.
Izoulet Jacques, 2005. Histoire d'une mission catholique dans le Pacifique au ${ }_{X I X}{ }^{e}$ siècle, Paris, L'Harmattan, 352p.

Kassovitz Mathieu, 1995. La Haine, 95 min, Christophe Rossignon, Kasso inc. Productions, Lazennec Productions.

—, 2000. Les rivières pourpres, 105 min, Production A. Goldman.

—, 2011. L'ordre et la morale, 136 min, Nord-Ouest Films, Christophe Rossignon.

La dépêche de Tahiti, quotidien de Polynésie française, juillet 2009 à décembre 2011.

LAFARgue Régis et Gilda Nicolau, 2003. La coutume judiciaire en Nouvelle-Calédonie, aux sources d'un droit commun coutumier, éd. Laboratoire de théorie du droit, Aix en Provence: Presses universitaires d'Aix-Marseille [en ligne] http://www.sudoc.fr/ 07416256X (Consulté le 17 octobre 2011).

Lallaour Mehdi, 2008. Retour sur Ouvéa, $70 \mathrm{~min}$, Mémoire Vives Productions.

LEBLIC Isabelle, 1993. Les Kanak face au développement, Grenoble, Presses universitaires de Grenoble, $412 \mathrm{p}$.

LegORJus Philippe, 1990. La morale et l'action, Paris : Fixot, $294 \mathrm{p}$.

—, 2011. La république et la morale, Paris, Plon

Les Nouvelles calédoniennes, quotidien de NouvelleCalédonie, juillet 2007 à décembre 2011.

MERLE Isabelle, 1995. Expériences coloniales. La Nouvelle-Calédonie (1853-1920), Paris, Belin.

MichalsKi Cédric, 2004. L'assaut de la grotte d'Ouvéa, analyse juridique, Paris, L'Harmattan, $320 \mathrm{p}$.

MuCKLE Adrian, 2010. La dernière révolte de Kanaky Nouvelle-Calédonie : vision de conflits passés dans un avenir commun, in Elsa Faugère et Isabelle Merle (éds), La Nouvelle-Calédonie, vers un destin commun?, Paris, Karthala, pp. 39-59.

NAEPELS Michel, 1998. Histoires de terres kanakes: conflits fonciers et rapports sociaux dans la région de Houailou (Nouvelle-Calédonie), Paris, Belin, $379 \mathrm{p}$.

_, 2010. Le devenir colonial d'une chefferie kanake (Houaïlou, Nouvelle-Calédonie), Annales. Histoire, Sciences sociales 4, pp. 913-943.

Plenel Edwy et Alain Rollat, 1988. Mourir à Ouvéa. Le tournant calédonien, Paris, La Découverte-Le Monde, $274 \mathrm{p}$.

SAnguinetti Antoine, Danielle Heldin, Guy RamiREZ, Violaine WeBEN et Jean-Pierre WeBEN, 1989. Enquête sur Ouvéa: rapports et témoignages sur

55. À ce sujet, Plenel et Rollat notent d'ailleurs : «Dans l'indifférence hexagonale, il s'est produit, ce 22 avril 1988, un événement inhabituel [...] : le gouvernement légal a confié à l'armée l'organisation, la conception et la mise en œuvre du maintien de l'ordre sur une partie du territoire national. [...] On ajoutera également la décision de déclarer provisoirement Ouvéa "zone militaire", comme on le fait de certains terrains de manœuvres. Le tout fera de l'atoll une zone à part dans la République française, interdite à la presse, privée de communications téléphoniques, coupée du reste du monde. » (Plenel et Rollat, 1988 : 126).

56. Jacques Chirac était alors Premier ministre de Françoise Mitterrand qui, en tant que président de la république et chef des armées, avait également donné son aval pour cette opération. 
les événements d'avril-mai 1988, Paris, Ligue des droits de l'Homme, Études et documentations internationales.

TANGOPI Benoît (09/11/2011), communiqué de presse.
Tuibaou Jean-Marie, 1996. Chap. 20. De l'art à la politique. Entretien avec Alain Plagne, le 6 mai 1985 à Paris, La présence kanak, Paris, Odile Jacob, pp. 191-207. 